\title{
Ultrasound Guided Laparoscopic Port Placement in a Patient with Congenital Anomaly of IVC Undergoing Sleeve Gastrectomy
}

\author{
Hatem Al-Saadi ${ }^{1}$ Sona Singh ${ }^{1} \cdot$ Kanagaraj Marimuthu $^{1} \cdot$ Alistair Sharples $^{1} \cdot$ Nagammapudur Balaji $^{1} \cdot$ Biju Thomas $^{1}$. \\ Vittal Rao ${ }^{1}$ (D)
}

Published online: 7 January 2020

(C) The Author(s) 2020

\begin{abstract}
We report a patient with obesity who underwent laparoscopic sleeve gastrectomy after pre-operative ultrasound mark up to enable safe port insertion due to presence of venous collaterals in the abdominal wall as a result of congenial IVC anomaly. This patient was falsely presumed to have NASH cirrhosis. Detailed preoperative workup ruled this out and led to the discovery of congenital IVC anomaly as the cause of engorged blood vessels in the anterior abdominal wall. On table ultrasound mark up of safe sites for port insertion enabled a safe laparosocpic sleeve gastrectomy on this patient.
\end{abstract}

Keywords Ultrasound · IVC Anomaly

\begin{tabular}{ll} 
Abbreviations \\
AF & Atrial fibrillation \\
BMI & Body mass index \\
CT & Computed tomography \\
DVT & Deep vein thrombosis \\
HT & Hypertension \\
IVC & Inferior vena cava \\
NASH & Non-alcoholic Steatohepatitis \\
NIDDM & Non-insulin-dependent diabetes mellitus \\
\hline
\end{tabular}

Vittal Rao

Vittal.Rao@uhnm.nhs.uk

Hatem Al-Saadi

hdhy@gmail.com

Sona Singh

Sona.Singh@uhnm.nhs.uk

Kanagaraj Marimuthu

Kanagaraj.Marimuthu@uhnm.nhs.uk

Alistair Sharples

Alistair.Sharples@uhnm.nhs.uk

Nagammapudur Balaji

Nagammapudur.Balaji@uhnm.nhs.uk

Biju Thomas

Biju.Thomas@uhnm.nhs.uk

1 Consultant Upper GI/Bariatric Surgeon, North Midlands Institute of Metabolic and Bariatric Surgery, University Hospital of North Midlands, Stoke-on-Trent, Staffordshire, UK

\author{
OSA Obstructive sleep apnea \\ PV Portal vein \\ USS Ultrasound scan
}

\section{Introduction}

Patients with obesity can have NASH with cirrhosis as one of the common comorbidities. They may present with prominent blood vessels in the abdominal wall if they have established portal hypertension (HT). We present an interesting case where the patient had prominent blood vessels in the abdominal wall with no associated portal HT. Investigative work-up revealed an atretic inferior vena cava (IVC) with collaterals as the cause of his engorged vessels in the abdominal wall. Preoperative ultrasound marking and guided port insertion enabled the patient to undergo a safe sleeve gastrectomy.

\section{Case Report}

A 52-year-old gentleman presented to the bariatric clinic for consideration of bariatric surgery. He had a BMI of 53.8 with a body weight of $160 \mathrm{~kg}$. He had significant comorbidities which included NASH, NIDDM, AF, OSA, and history of recurrent DVT.

He had history of recurrent DVT and chronic venous insufficiency of both legs resulting in chronic venous ulcers since 
Fig. 1 CT demonstrating venous collaterals arising from the common iliac veins on both sides and running along the flanks to the azygos and just below the skin in the subcutaneous plane
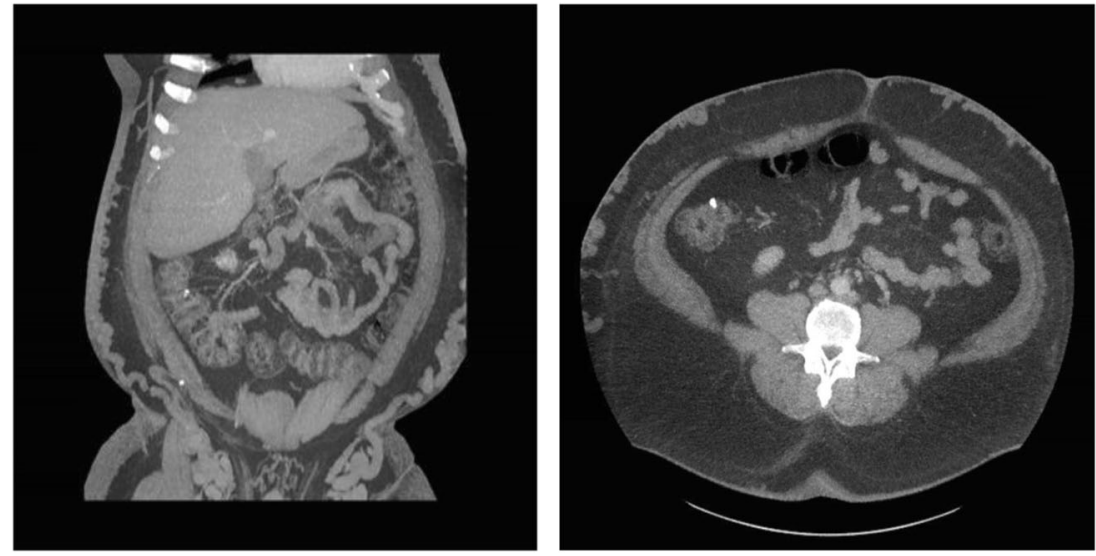

the age of 20. Clinical examination revealed distended veins in the anterior abdominal wall and both flanks and was presumed to have portal HT secondary to NASH Cirrhosis. However, an upper GI endoscopy ruled out varices, and an USS abdomen revealed NASH with no evidence of cirrhosis and a normal PV flow.

He had a CT scan which revealed numerous venous collaterals in the anterior and lateral abdominal wall in the subcutaneous plane distributed symmetrically, and this appeared to originate from both the groins. The IVC was small in calibre measuring $1.7 \mathrm{~cm}$ in maximal axial dimension and appeared to be atretic. There was no evidence of IVC thrombosis (Fig. $1)$.

The patient was counselled for laparoscopic sleeve gastrectomy. He underwent on table preoperative US-guided mapping of the anterior abdominal wall to guide port placement with a view to avoid injury to the venous collaterals (Fig. 2). The port sites were slightly deviated from the standard port positions, and the patient underwent standard laparoscopic sleeve gastrectomy. He made a good recovery and is doing well on post-op follow-up.

\section{Discussion}

Congenital anomalies of the IVC are not uncommon with significant variations due to the complexities around its development during embryogenesis [1]. Awareness of these anomalies is important to avoid pitfalls in management of these patients as they are mostly asymptomatic.

One of the rare presentations of patients with congenital anomalies of the IVC is DVT at a young age [2]. A recognized variant is the absence or atretic infra-renal IVC as is the case in our patient. This results in development of collaterals from the iliac veins draining along the abdominal wall into azygos and hemiazygos vein. There is controversy as to whether this is a true embryonic anomaly or whether it is the result of perinatal IVC thrombosis [3].

It is suggested that the venous return in the case of an absent or atretic IVC is suboptimal in spite of collaterals and hence results in chronic venous hypertension in the lower limbs leading to chronic venous insufficiency and venous ulcers as was the case in our patient.
Fig. 2 Pre-op ultrasound-guided marking of port sites prior to laparoscopic sleeve gastrectomy
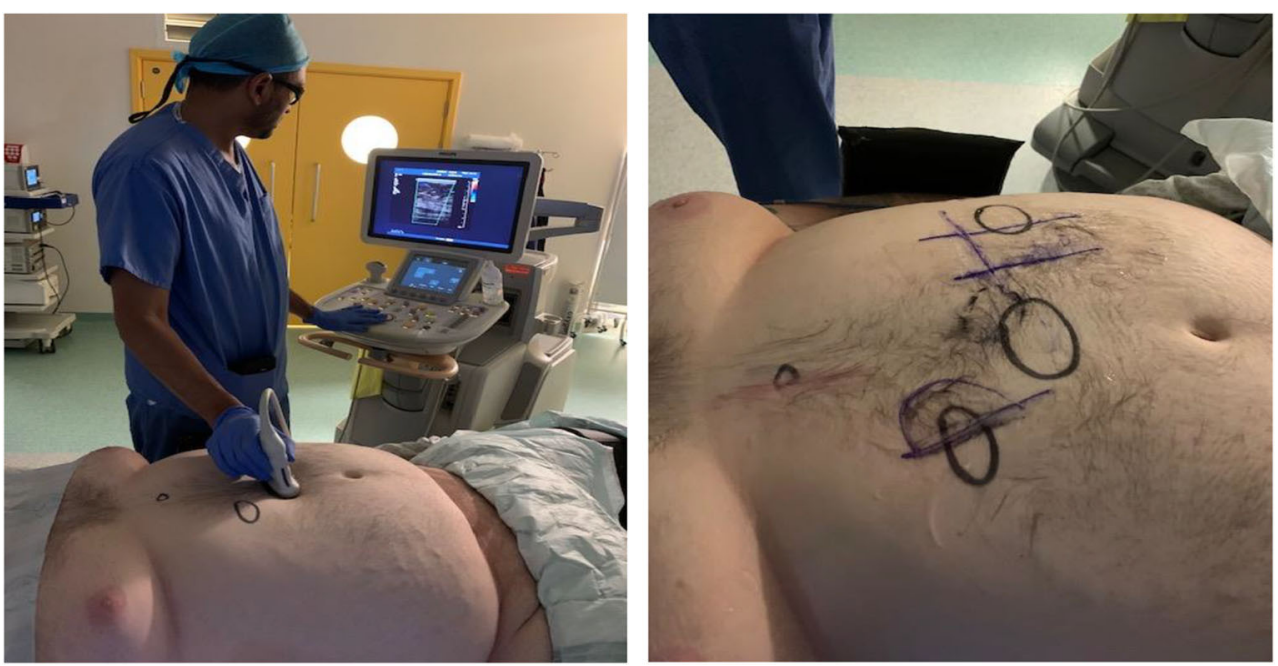
CT is a very reliable noninvasive imaging modality in diagnosing IVC anomalies. This rules out other causes of distended venous collaterals in the abdominal wall, especially those associated with portal HT in the background of NASH [4].

This is the first reported case in literature where any form of laparoscopic surgery has been done in a patient with engorged blood vessels in the anterior abdominal wall due to atretic IVC. This is certainly the first patient with morbid obesity who had laparoscopic sleeve gastrectomy. With preoperative ultrasound marking, the surgery can be done in a safe manner as has been demonstrated in our patient.

\section{Conclusion}

Congenital malformations of the IVC are rare. Most patients are asymptomatic, and the anomalies are picked up during radiological investigations. Knowledge of these congenital anomalies is important prior to performing any operative/ laparoscopic surgery and especially in patients with obesity and associated NASH as it could be potentially labelled as part of the picture of portal HT and the patients being denied bariatric surgery. Preoperative ultrasound-guided placement of ports enables any laparoscopic procedure including bariatric surgery to be done safely as has been demonstrated in this patient.

Acknowledgments Mr. Al-Saadi and Dr. Singh collected the data and were involved with the write up. Mr. Marimuthu, Mr. Balaji, and Mr. Sharples offered editorial support. Dr. Thomas and Mr. Rao were involved with the care of the patient and overall supervision of the project.

\section{Compliance with Ethical Standards}

Conflict of Interest The authors declare that they have no conflict of interest.
Informed Consent Informed consent was obtained from the patient for writing up this article and including images.

Open Access This article is licensed under a Creative Commons Attribution 4.0 International License, which permits use, sharing, adaptation, distribution and reproduction in any medium or format, as long as you give appropriate credit to the original author(s) and the source, provide a link to the Creative Commons licence, and indicate if changes were made. The images or other third party material in this article are included in the article's Creative Commons licence, unless indicated otherwise in a credit line to the material. If material is not included in the article's Creative Commons licence and your intended use is not permitted by statutory regulation or exceeds the permitted use, you will need to obtain permission directly from the copyright holder. To view a copy of this licence, visit http://creativecommons.org/licenses/by/4.0/.

\section{References}

1. Edward Bass J, Redwine MD, Kramer LA, et al. Spectrum of congenital anomalies of the inferior vena cava : Cross sectional imaging findings. Radiographics. 2000:20(3). https://doi.org/10.1148/ radiographics.20.3.g00ma09639.

2. Chee YL, Dominic J, Watson CG, et al. Inferior vena cava malformation as a risk factor for deep venous thrombosis in the young. Br J Haematol. 2001;114:878-80. https://doi.org/10.1046/j.1365-2141. 2001.03025.x.

3. Tharumenthiran R, Hughes TMD, Richardson AJ. Perinatal inferior vena cava thrombosis and absence of the infrarenal inferior venacava. J Vasc Surg. 2001;33:1097-9. https://doi.org/10.1067/ mva.2001.114205.

4. Ueda J, Hara K, Kobayashi Y, et al. Anomaly of the inferior vena cava observed by CT. Comput Radiol. 1983;7(3):145-54. https://doi. org/10.1016/0730-4862(83)90089-6.

Publisher's Note Springer Nature remains neutral with regard to jurisdictional claims in published maps and institutional affiliations. 